\title{
Observation of Landau levels at the InAs(110) surface by scanning tunneling spectroscopy
}

\author{
J. W. G. Wildöer \\ Research Institute for Materials, University of Nijmegen, Toernooiveld, 6525 ED Nijmegen, The Netherlands \\ and Department of Applied Physics, Delft University of Technology, P.O. Box 5046, 2600 GA Delft, The Netherlands \\ C. J. P. M. Harmans \\ Department of Applied Physics, Delft University of Technology, P.O. Box 5046, 2600 GA Delft, The Netherlands \\ H. van Kempen \\ Research Institute for Materials, University of Nijmegen, Toernooiveld, 6525 ED Nijmegen, The Netherlands
}

(Received 28 March 1997)

\begin{abstract}
Low-temperature scanning tunneling spectroscopy measurements on clean InAs(110) surfaces demonstrate the possibility to investigate Landau quantization with subnanometer resolution. Separate Landau levels are resolved at magnetic fields of $2 \mathrm{~T}$ and larger. Experiments with different tips show a significant tip dependence. In zero field resonances are observed near the onset of the conduction band, which are attributed to tip-induced band bending. Although Landau quantization is only present parallel to the sample surface, the Landau levels give a large contribution to the total tunnel current. [S0163-1829(97)52424-6]
\end{abstract}

Recently, several experiments ${ }^{1-5}$ have demonstrated the possibility to use scanning tunneling microscopy to investigate the spatial behavior of the conduction and valence bands in the vicinity of distortions in the electrostatic potential. The edges of these bands determine the transport properties of a semiconductor, and therefore the possibility to investigate the energy bands spatially resolved can be of great value. In semiconductors, the Fermi wavelength and also the screening length exceed the atomic scale. As a consequence dopants just below the surface can influence the carrier density at the surface. Johnson et al. ${ }^{1}$ and Zheng et al. ${ }^{2}$ have shown that these local variations in the band bending appear as hillocklike features in scanning tunnel microscope (STM) images of the $\operatorname{GaAs}(110)$ surface, superimposed on the atomic sublattice (see also Ref. 3). On III-V semiconductor heterostructures Feenstra et al. ${ }^{4}$ were able to resolve the subband energies of a superlattice and to characterize the roughness of the interfaces ${ }^{5}$ by scanning tunneling spectroscopy. In their experiments the measurements were performed at room temperature on clean cross-sections of the heterostructure after it was cleaved under UHV conditions.

In this paper we demonstrate that scanning tunneling microscopy also allows the direct, spatially resolved observation of the quantization of conduction electrons into Landau levels in an external magnetic field. Since the energy scales of Landau-level quantization are much smaller than those related to dopants and heterostructures, the STM in our experiments is operated at $4 \mathrm{~K}$, which yields a significant increase in the spectral resolution $\left(k_{B} T \sim 0.4 \mathrm{meV}\right.$ at $\left.4 \mathrm{~K}\right)$ compared to room temperature. The enhanced resolution also enables us to examine the onset of the conduction band in the $I-V$ curves in zero field in more detail. In our measurements on clean $\operatorname{InAs}(110)$ surfaces we observe sharp resonances that are strongly tip dependent.

The opportunity to investigate Landau levels with the lateral resolution of an STM opens interesting experimental prospects. Transport properties in two-dimensional electron gases subjected to Landau quantization are highly anomalous. The most famous example is the quantum Hall effect, discovered by von Klitzing, Dorda, and Pepper in $1980 .^{6}$ During the last decade it has become clear that the spatial evolution of the Landau levels near the edges of a twodimensional electron gas (2DEG) leads to the formation of so-called edge states, ${ }^{7}$ which are responsible for many of the transport phenomena. Recently these edge states have been imaged to a lateral resolution of a few micrometers, using optical means. ${ }^{8}$ Imaging the Landau levels with nanometer resolution could yield detailed information about sizes and shapes of the edge channels and allow comparison to selfconsistent theories. ${ }^{9}$

For our experiments we have chosen InAs because of its small effective electron mass at the conduction band edge, $m_{e f f}=0.023 m_{e}$, resulting in a significant Landau-level splitting already at low magnetic fields. Also, in the presence of Fermi-level pinning, surfaces of InAs can contain surface 2DEG's. ${ }^{10-12}$ InAs is a small band-gap semiconductor, $E_{g}=350 \mathrm{mV}$, and as most IIII-V semiconductors, has a zincblende structure.

The electronic properties of InAs surfaces have been studied by various other techniques. The existence of a surface 2DEG, due to surface pinning of the Fermi level in the conduction band, was for the first time indicated in transport measurements by Kawaji and Gatos. ${ }^{10}$ Tsui investigated the $n$-type InAs accumulation layer by planar tunneling spectroscopy and was able to directly determine the energies of the Landau levels, both in the surface 2DEG and in the bulk conduction band. ${ }^{11,12}$ The planar method used by Tsui is based on the same principles as tunneling spectroscopy with an STM. In the planar geometry usually an oxide is used as an insulator between the two electrodes, whereas in an STM geometry the vacuum barrier separates the electrodes. The use of the vacuum barrier and the lateral scanning capability, however, allows us to discriminate between contributions 


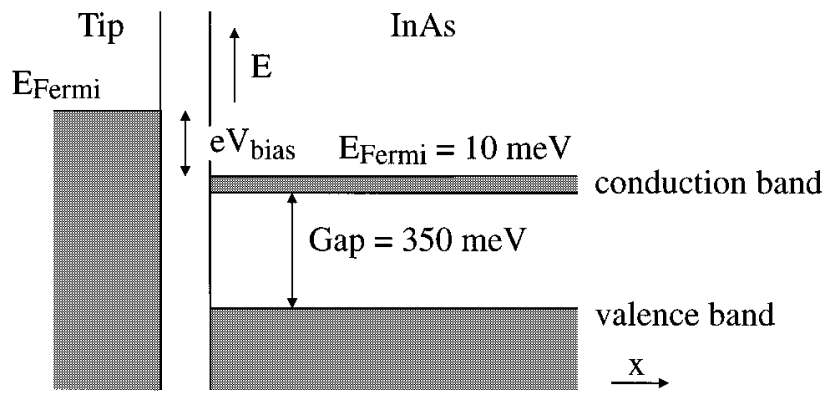

FIG. 1. Energy diagram of the tip-vacuum-InAs system. Bias voltages are applied to the sample relative to the tip. At positive voltages electrons tunnel from the tip into the InAs conduction band. The InAs is degenerate, with a Fermi energy of $10 \mathrm{meV}$ and a band gap of $350 \mathrm{mV}$.

from contaminants and defects versus those of the actual surface and also opens the possibility to obtain spatially resolved information.

The experimental objective here has been to observe Landau levels at the semiconductor surface with an STM. For this we have prefered to investigate clean surfaces without deliberate Fermi-level pinning. In order to establish these surfaces we have performed our experiments with a lowtemperature $\mathrm{STM}^{13}$ equipped with an in situ cleaver. The STM is mounted in a cryostat, inside the bore of a superconducting $10 \mathrm{~T}$ magnet. At liquid He temperatures the vapor pressure of oxygen is extremely low $\left(<10^{-15}\right.$ Torr). Surfaces of III-V compound semiconductors, which normally oxidize very quickly, will stay free of contaminants for many days under these conditions. The InAs used in our experiments is lightly $n$ doped, $n=2.510^{16} \mathrm{~cm}^{-3}$, yielding a Fermi energy of $10 \mathrm{meV}$. This doping concentration ensures a finite bulk conductivity at low temperatures, irrespective of the presence of a surface 2DEG. All the measurements presented here are performed with $\operatorname{Pt}(90 \%) \operatorname{Ir}(10 \%)$ tips, cut in ambient with scissors. The bias voltages are applied to the sample relative to the tip. This implies that at positive voltages the Fermi level of the tip is lined up in the conduction band of the InAs. A schematic energy diagram is shown in Fig. 1.

On surfaces that were cleaved in situ at low temperatures, atomic resolution was easily achieved. Depending on the po- larity of the bias voltage either the In or the As atoms are imaged. The images are similar to those obtained by Feenstra et al. on GaAs (Ref. 14) and depend on the polarity of the bias voltage. Spectroscopy curves on the clean InAs(110) surfaces give a band gap of $0.50 \pm 0.10 \mathrm{~V}$, larger than the expected value of $0.35 \mathrm{~V}$. The deviation is attributed to tip induced band bending. We omit the discussion of the largescale spectroscopic features here (see Ref. 15) and focus on some of the details that are revealed because of the enhanced spectroscopic resolution.

In the majority of our experiments we observed sharp features in the $d I / d V$ near the onset of the conduction band [Figs. 2(a) and 2(b)]. These features are not due to defects or contaminants at the surface, since by topography it is assured that spectra are taken on flat and clean terraces, tens of nanometers away from any irregularity present at the surface. The occurrence and the energies of these sharp resonantlike features is tip dependent. With a particular tip, $I-V$ curves are identical over the whole surface (with the exception of areas with steps and defects).

Figure 2(c) shows $d I / d V$ curves obtained with magnetic fields of $3 \mathrm{~T}$ and $4 \mathrm{~T}$ applied perpendicular to the surface. Besides the peaks below the conduction band edge, which were already present at zero field, oscillations in the $d I / d V$ at higher voltages are present. The period of the oscillations scales with the applied magnetic field. From Fig. 2(c) we determine the periods to be $\sim 12 \mathrm{mV}$ at $3 \mathrm{~T}$ and $\sim 16 \mathrm{mV}$ at $4 \mathrm{~T}$. These values are of the same order as the expected Landau-level splittings $\left(\hbar B / m_{e f f}\right)$ of $15 \mathrm{mV}$ and $20 \mathrm{mV}$ (with $m_{e f f}=0.023 m_{e}$ ). We therefore conclude that the oscillations in the $d I / d V$ are due to Landau quantization in the plane of the sample surface. An important question that has to be addressed is whether the oscillations originate from bulk electrons or from a possible surface 2DEG.

In Fig. 3(a) the voltages at which the oscillation maxima occur are shown as a function of the Landau-level number $l$ for different magnetic fields. ${ }^{16}$ From this figure we can clearly distinguish two regimes: one where the Landau-level separation is nearly constant, and another where the separation is not constant and where the voltages corresponding with the maxima of the oscillations are tip dependent.

In the bulk conduction band the Landau-level separation is constant and scales with magnetic field. From the oscilla-
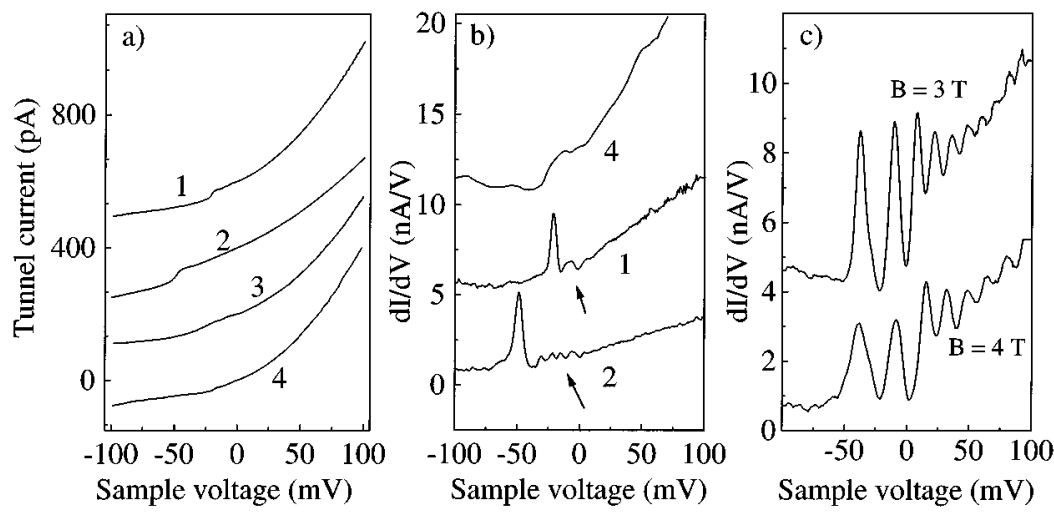

FIG. 2. (a) $I-V$ spectra near the onset of the conduction band. The resonances in the $d I / d V$ (b) are reproducible and independent of position (also the small ones indicated by the arrows). In some cases no resonances are observed. The curves are offset for clarity. (c) $d I / d V$ at $B=3 \mathrm{~T}$ and $B=4 \mathrm{~T}$. Oscillations at positive sample voltages scale with magnetic field. The $B=3 \mathrm{~T}$ curve is shifted by $4 \mathrm{nA} / \mathrm{V}$ for clarity. 

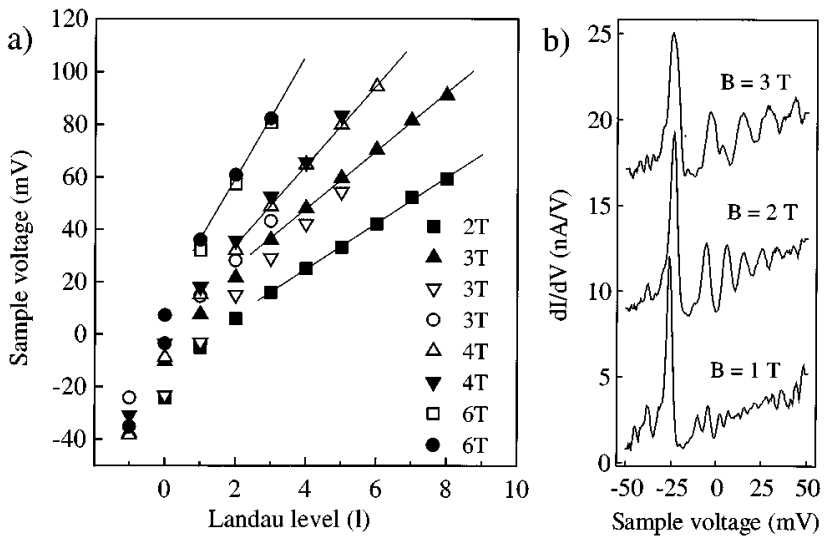

FIG. 3. (a) The maxima in the $d I / d V$ as a function of the Landau-level number $l$. Measurements at the same magnetic field, but with different tips, are indicated by different symbols. (b) Landau levels at low magnetic fields measured with the same tip. The peak at $-25 \mathrm{mV}$ does not split into seperate levels when the magnetic field is increased, and also it does not shift to higher energies. The two upper curves are offset for clarity.

tion period (solid line fits) we obtain an effective electron mass of $0.028 \pm 0.003 m_{e}$. This value is larger than the one known for the conduction band minimum $\left(0.023 m_{e}\right)$. In our measurements the oscillation period is, however, obtained between 40 and $100 \mathrm{meV}$ above the conduction band edge, where the effective electron mass is expected to be slightly higher due to the nonparabolicity of the band edge. A similar increase of $m_{\text {eff }}$ has been observed by Tsui ${ }^{11}$ through planar InAs-oxide- $\mathrm{Pb}$ junctions.

At the lower voltages the positions of the maxima of different measurements do not coincide. As can be seen in Fig. 3(a), a different tip results in a shift of the low bias voltage maxima to higher or lower energies. The peaks in the $d I / d V$ below the bulk conduction band edge do not show a dependence on the applied magnetic field. Figure 3(b) shows spectra obtained with the same tip at low magnetic fields. The first peak at $-25 \mathrm{mV}$ does not shift significantly and also does not give rise to separate Landau levels . The oscillations observed at larger bias voltages are all situated in the bulk conduction band. If the first peak would be due to a surface 2DEG, we would expect additional peaks with a separation of $\hbar B / m_{e f f}$ and also a shift of the first peak equal to half this period. We therefore conclude from Fig. 3(b) that the peaks at negative sample voltage should not be attributed to a surface $2 \mathrm{DEG}$, and that such a $2 \mathrm{DEG}$ is not present at the investigated surface. We will discuss the origin of the negative bias peaks below.

On atomically flat InAs(110) surfaces all surface atoms carry one dangling bond. The In dangling bonds have an energy in the conduction band $(1.3 \mathrm{eV}$ above the conduction band minimum), and the As states have an energy in the valence band $\left(-1.2 \mathrm{eV}\right.$ below the valence band maximum). ${ }^{17}$ So, since the band gap is free of intrinsic surface states, a surface 2DEG could only be expected through Fermi-level pinning at the surface. The in situ cleaved surfaces are practically free of contaminants and therefore the absence of a surface 2DEG is in accordance with what is expected.

Figures 2(c) and 3(b) show that the Landau levels are responsible for $20-50 \%$ of the total tunnel current. With the magnetic field applied perpendicular to the sample surface the quantization of Landau levels is present only parallel to the surface. In the direction perpendicular to the surface a continuous density of states should persist. Our measurements indicate that a significant fraction of the tunnel current is carried by states parallel to the sample surface.

We now discuss the role of the tip in our measurements. In Fig. 3(a) different tips result in different bias voltage position of the peaks, in particular at low bias voltages. There are several tip-dependent parameters that can influence the spectra. Every tip is characterized by a unique transfer Hamiltonian matrix, ${ }^{18}$ which is determined by the outer-most atoms of the tip. The transfer Hamiltonian matrix defines which states have the largest probability for tunneling between tip and sample. Second, the work function difference between tip and sample, together with the applied bias voltage, determines the magnitude of the local electric field in the tip region, and therefore the local density of states. The spatial profile of the electric field depends on a third tipdependent parameter, namely the geometric shape of the tip.

Since the Landau levels at higher energies appear to be less sensitive to tip changes than those at lower electron densities [Fig. 3(a)], the peak voltage position shifts have to be attributed to differences in the local electric field. This suggests that every PtIr tip has a different work function $W_{t i p}$. Tip changes resulting in a shift of Landau-level positions were occasionally also observed during the experiments.

For the work function of the InAs(110) surface, $W_{\text {InAs }}$, a value of $4.9 \mathrm{eV}$ has been reported. ${ }^{19} \mathrm{We}$ estimate a value of the order of $5.5 \mathrm{eV}$ (Ref. 20) for PtIr. Work functions are however critically sensitive to surface conditions and the surface structure. ${ }^{21}$ This sensitivity can account for the different energies of the Landau levels with different tips.

The sharp peaks in Fig. 2(b) and in the negative bias voltage range in Figs. 2(c) and 3(b) are situated at energies below the bulk conduction band edge. We therefore conclude that in these measurements an accumulation layer is formed instead of the anticipated depletion layer. Since we did not find any evidence for a magnetic field dependence of peaks at negative bias voltages, the accumulation layer is only present in the vicinity of the tip, and in fact forms a small accumulation dot. The electrons in the dot locally screen the electric field originating from the tip. The formation of an accumulation dot below the tip leads to the conclusion that in many of our experiments the work function of the tip is lower than that of the InAs(110) surface. Depending on the work function difference between tip and sample, and the spatial profile of the electric field, one or more electrons will be present. When the Fermi energy of the tip is decreased, the localized states of the dot will become available for tunneling (Fig. 4). With every different state the tunnel current increases stepwise, giving a peak in the $d I / d V$. To understand the variations in height of the various peaks or the small resonances indicated by the arrows in Fig. 2(b), a more detailed model is required. For example, the presence of electrons in the induced dot will rearrange the electrons in the bulk conduction band at the surface, influencing their probability to tunnel to the tip. The small resonances of Fig. 2(b) may well be explained by taking into account quantum size effects. 
a)

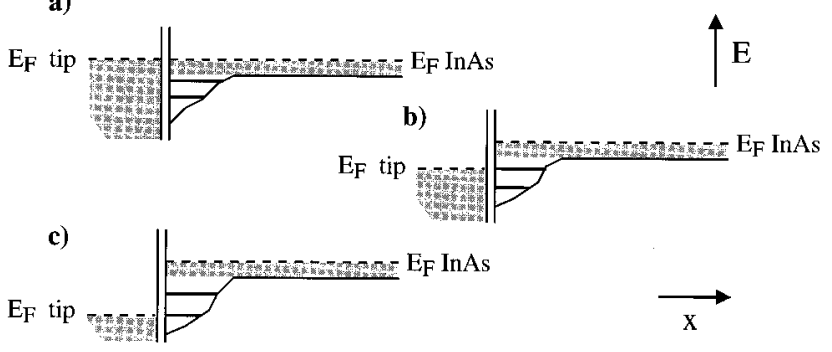

FIG. 4. Tip induced localized states contribute to the tunnel current when the Fermi level of the tip is decreased. In (b) and (c) the Fermi level equals the energy of one of the localized states. The energies of the bound states vary with the applied bias voltage.

Our model assumes $W_{\text {tip }}<W_{\text {InAs }}$, opposite to the expected $W_{t i p}>W_{\text {InAs }}$. In our setup tips can not be prepared in situ, and are cut in ambient with scissors. Before cutting the tip, scissors and PtIr wire are cleaned extensively with isopropyl alcohol. Furthermore, the PtIr is cut in such a way that the tip is composed of material inside the wire. Realistically however, contamination of the tip surface due to exposure to air can not be avoided, leading to a lower work function of the tip. For heat exchange purposes a small amount of $\mathrm{He}$ is present in the vacuum can during the experiments. Although $\mathrm{He}$ is an inert gas its presence at the tip and the sample surface is likely to influence both work functions. ${ }^{22,23}$

In conclusion, we have performed tunneling spectroscopy measurements with an STM on clean InAs(110) surfaces at low temperatures. Landau levels have been observed by scanning tunneling spectroscopy. The strongly enhanced energy resolution at low temperature allows the investigation of $I-V$ 's on semiconductor surfaces in significantly more detail than at room temperature. This enables to resolve separate Landau levels already at relatively low magnetic fields. Our data show that every tip uniquely influences the spectra near the onset of the conduction band. At zero field sharp resonances are observed that are attributed to the formation of a "dot" via tip-induced accumulation at the $\operatorname{InAs}(110)$ surface.

The absence of surface states near the band gap results in measurements that are strongly tip dependent. To reduce the influence of the electric field of the tip the Fermi level has to be pinned at the semiconductor surface. Fermi-level pinning can be realized for example by depositing a thin layer of adsorbates on the surface ${ }^{24}$ or by cleaving the semiconductor in such a way that a large density of steps is obtained at the surface. $^{25}$ To establish a surface two-dimensional electron gas the Fermi level at the surface has to be pinned in the conduction band.

The Landau-level measurements show that a significant fraction of the tunnel current is carried by states parallel to the sample surface. Landau levels at semiconductor surfaces may provide a convenient system to investigate $k$ conservation in the STM tunnel geometry.

This work was supported by the Dutch Foundation for Fundamental Research of Matter FOM We gratefully acknowledge M.C.M.M. van der Wielen and R. Jansen for useful discussions and A.J.A. van Roij for technical assistance.
${ }^{1}$ M.B. Johnson et al., Appl. Phys. Lett. 63, 2923 (1993).

${ }^{2}$ J.F. Zheng et al., Phys. Rev. Lett. 72, 1490 (1994).

${ }^{3}$ M.C.M.M. van der Wielen, A.J.A. van Roij, and H. van Kempen, Phys. Rev. Lett. 76, 1075 (1996).

${ }^{4}$ R.M. Feenstra, D.A. Collins, D.Z.-Y. Ting, M.W. Wang, and T.C. McGill, Phys. Rev. Lett. 72, 2749 (1994).

${ }^{5}$ See also W. Wu et al., J. Vac. Sci. Technol. A 13, 602 (1995).

${ }^{6}$ K. v. Klitzing, G. Dorda, and M. Pepper, Phys. Rev. Lett. 45, 494 (1980).

${ }^{7}$ M. Büttiker, in Nanostructured Systems, edited by Mark Reed (Academic Press, Orlando, Florida, 1992), p. 191.

${ }^{8}$ R.J.F. van Haren, F.A.P. Blom, and J.H. Wolter, Phys. Rev. Lett. 74, 1198 (1995).

${ }^{9}$ D.B. Chklovskii, B.I. Shklovskii, and L.I. Glazman, Phys. Rev. B 46, 4026 (1992).

${ }^{10}$ S. Kawaji and H.C. Gatos, Surf. Sci. 7, 215 (1967).

${ }^{11}$ D.C. Tsui, Phys. Rev. B 4, 4438 (1971).

${ }^{12}$ D.C. Tsui, Phys. Rev. B 8, 2657 (1973).

${ }^{13}$ J.W.G. Wildöer, A.J.A. van Roij, H. van Kempen, and C.J.P.M. Harmans, Rev. Sci. Instrum. 65, 2849 (1994).

${ }^{14}$ R.M. Feenstra, J.A. Stroscio, J. Tersoff, and A.P. Fein, Phys. Rev. Lett. 58, 1192 (1987).

${ }^{15}$ R.M. Feenstra, Phys. Rev. B 50, 4561 (1994).
${ }^{16}$ The labeling of the peaks by the Landau-level numbers $l$ is somewhat arbitrary. We extended the solid line fits to low voltages and assigned the Landau-level numbers in such a way that the 0th Landau level would be just above $V_{\text {Sample }}=-10 \mathrm{mV}$ (the bottom of the conduction band). The measured Landau-level positions are influenced by tip-induced band bending.

${ }^{17}$ J. van Laar, A. Huijser, and T.L. van Rooy, J. Vac. Sci. Technol. 14, 894 (1977).

${ }^{18}$ C.J. Chen, Introduction to Scanning Tunneling Microscopy (Oxford University Press, Oxford, 1993).

${ }^{19}$ G.W. Gobeli and F.G. Allen, Phys. Rev. 137, 245 (1965).

${ }^{20}$ D.R. Lide, CRC Handbook of Chemistry and Physics, 72nd ed. (CRC Press, Boca Raton, 1991).

${ }^{21}$ For example: Solid State Surface Science, edited by M. Green (Marcel Dekker, New York, 1969).

${ }^{22}$ See for example: J. Patigny, Y. Barbaux, and J.-P.A. Beaufils, in Adsorption-desorption Phenomena, edited by F. Ricca (Academic Press, New York, 1994), p. 49, and references therein.

${ }^{23}$ The presence of helium has been found to influence the tunnel current between two metal electrodes: R.J.P. Keijsers et al., Phys. Rev. Lett. 76, 1138 (1996).

${ }^{24}$ R.M. Silver, J.A. Dagata, and W. Tsjeng, J. Appl. Phys. 76, 5122 (1994).

${ }^{25}$ A. Vaterlaus et al., J. Vac. Sci. Technol. B 11, 1502 (1993). 